\title{
Preface: Human Immunology at SIgN
}

\author{
Philippe Kourilsky $\cdot$ Paola Ricciardi-Castagnoli
}

Published online: 7 April 2012

(C) Springer Science+Business Media, LLC 2012

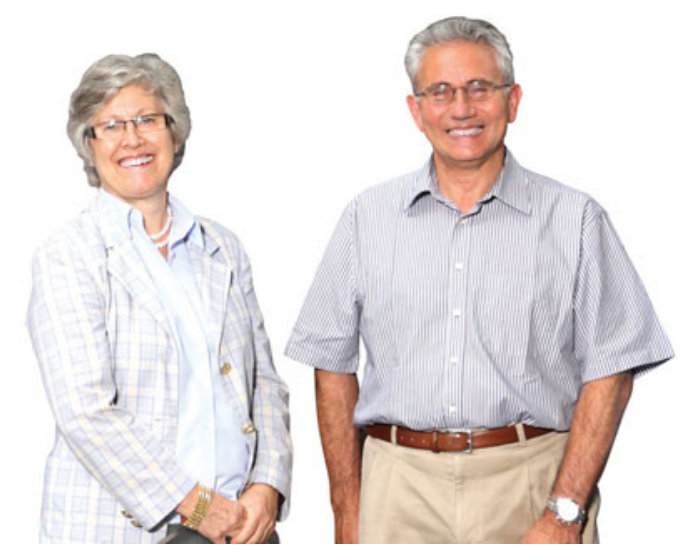

Paola Ricciardi-Castagnoli Philippe Kourilsky
The Singapore Immunology Network (SIgN) was launched a few years ago by the governmental Agency for Science, Technology, and Research (A*STAR), with the aim of developing immunology research in Singapore and the surrounding region in Asia. The SIgN research centre is located in Biopolis, a research hub created at the end of 2003, largely under the influence of Sidney Brenner, who advised the Singapore government at the time.

Immunology was one of the latest additions. The strategic decision was made in early 2006 that SIgN would be focused on Human Immunology. It was anticipated that cutting-edge technologies would held driving the research and that the choice of working mostly on humans would generate bridges between academic, clinic, and industrial partners. SIgN laboratories opened in early 2008. Since then, SIgN has grown rapidly to 200 investigators coming from 27 different countries of the world, working under 25 renowned Principal Investigators (see: www.sign.a-star.edu.sg).

SIgN's mandate is to build up a strong platform in Human Immunology research for better translation of the research findings into clinical applications, to address the many unmet medical needs, and contribute to the development of innovation in Singapore. For that purpose, we have established a highly integrated approach using a number of state-of-the art technological platforms such as functional genomics and bioinformatics, functional imaging and flow cytometry, immunomonitoring and proteomics.

P. Kourilsky · P. Ricciardi-Castagnoli ( $\square)$

Singapore Immunology Network (SIgN), Agency for Science,

Technology and Research (A*STAR), 8A Biomedical Grove,

\#04 Immunos, Biopolis, Singapore 138648, Singapore

e-mail: paola_castagnoli@immunol.a-star.edu.sg

P. Kourilsky

e-mail: philippe_kourilsky@immunol.a-star.edu.sg
In practice, the system has been designed in such a way as to allow us to examine large cohorts of patients. The platforms are now used to discover correlates of protection, new biomarkers, and novel molecular targets in infectious diseases such as TB, malaria, or dengue as well as in other immuno-related diseases. In addition, we have created a high throughput platform for the generation of fully human monoclonal antibodies to define new epitopes of infectious agents, design better vaccines, and develop novel therapeutic antibodies.

Other areas of research in SIgN include inflammatory diseases such as rheumatoid arthritis and gout, as well as the tumor microenvironment of solid tumors and their immune infiltrates. Immunity to lipid antigens is also studied in autoimmune diseases as well as in TB. Finally, we have recently initiated a program to investigate allergies, which is a growing medical need in Singapore.

In this very short time, SIgN has implemented a clear scientific strategy to pursue basic research excellence coupled to applied research programs to discover new targets for immuno-related human diseases. Thanks to our extensive and growing network of clinical and industrial partners, we hope that the knowledge we produce will translate into novel ways of solving urgent and unmet medical needs.

Acknowledgments We would like to acknowledge Barbora Fricova's excellent editorial work in assembling and coordinating this special volume devoted to SIgN. We are also very grateful to Lucy Robinson and Neil McCarthy of Insight Editing London for their dedicated and diligent revision of the manuscripts. Finally, we wish to thank A*STAR for financially supporting the research performed by SIgN. 